\title{
STABLE EQUILIBRIA UNDER THE TWO-ISLAND MODEL
}

\author{
M. G. BULMER \\ Department of Biomathematics, University of Oxford
}

Received 23.ix.70

\section{INTRODUCTION}

The following problem has been considered by Moran (1962, pp. 173-5). Suppose that there are two large populations of the same constant size, but subject to different selection pressures, and that after selection a proportion $m$ migrates from each population to the other one and that mating then occurs at random. Consider a character determined by a single locus with two alleles, $C_{1}$ and $C_{2}$, and suppose that the relative fitnesses of the three genotypes, $C_{1} C_{1}, C_{1} C_{2}$ and $C_{2} C_{2}$, are $1+s_{1}, 1$ and $1-s_{1}$ in the first population and $1+s_{2}, 1$ and $1-s_{2}$ in the second population. Moran finds that if selection acts in the opposite direction in the two populations, so that $s_{2}=-s_{1}$, and if $m<\frac{1}{2}$, then there is always a non-trivial stable equilibrium. Moran summarises his conclusions as follows: "So long as selection operates in opposite directions in the two subpopulations a stable polymorphism is possible. This is very similar to the situation in a single population in which the heterozygote is more favoured than either of the homozygotes."

In many situations the above model, which may be called the two-island model, provides a more plausible mechanism for generating a stable equilibrium and hence maintaining genetic variability than the frequently invoked explanation of heterozygote advantage in a single, panmictic population. The model can be generalised in several ways, either by extending it to more than two populations or by dropping the requirements of symmetry in the selection coefficients and migration rates (see, for example, Levene, 1967). The purpose of this paper is to extend the model to a metric character determined by a relatively large number of loci with individually small effects and subjected to selection for different optimal values in the two populations. The methods used depend extensively on results obtained in a previous paper for a single, panmictic population (Bulmer, 1971); as in that paper the effects of linkage disequilibrium will be ignored on the grounds that they are likely to be small under the rather weak selective pressures and low migration rates likely to be found under natural conditions. We shall begin by formulating the two-island model in the simple case when there is no dominance. The reader who is not interested in the mathematical derivation of the results may like to read the discussion first.

\section{Formulation of the tWo-ISLAND MOdel}

Consider a metric character, $y$, whose genetic component is determined by $\mathcal{N}$ loci with equal effects. We shall suppose that each locus has two alleles, $C_{1}$ and $C_{2}$, and that the effects of the three possible genotypes, $C_{1} C_{1}, C_{1} C_{2}$ and $C_{2} C_{2}$, are $-a, a D$ and $a$ respectively, where $D$ is a measure of the degree and direction of dominance; for the moment it will be assumed that $D=0$. 
If the frequency of the $C_{1}$ allele at the $i$ th locus is $p_{i}$, then the genetic variance is

$$
V_{G}=2 a^{2} \sum_{i=1}^{N} p_{i} q_{i}=2 a^{2} \mathcal{N}\left(\overline{p_{i} q_{i}}\right)
$$

where $\left(\overline{p_{i} q_{i}}\right)$ is the average value of $p_{i} q_{i}$ over the different loci. If the genetic variance is to remain approximately the same as the number of loci increases, then $a^{2} \mathcal{N}$ must be approximately constant. We shall therefore regard $a^{2}$ as a quantity of order $1 / \mathcal{N}$, and we shall ignore all terms of smaller magnitude. We shall also suppose that there is an independent, normally distributed environmental component of $y$.

Suppose now that there are two large populations of the same constant size, but subject to different selection pressures, and that after selection a proportion $m$ migrates from each population to the other one and that mating then occurs at random. If the frequency of the $C_{1}$ allele at the $i$ th locus in the first population is $p_{1 i}$ at the zygote stage, then the change in the gene frequency as a result of selection is, to order $a^{2}$ and hence to order $1 / \mathcal{N}$,

$$
\Delta p_{1 i}=p_{1 i} q_{1 i}\left\{A_{1} a-\frac{1}{2} B_{1} a^{2}\left(p_{1 i}-q_{1 i}\right)\right\}
$$

with a corresponding equation for $\Delta p_{2}$, the change in the gene frequency in the second population, where the coefficients $A_{1}$ and $B_{1}$ depend on the selection pressure and on the distribution of $y$ in the first population (Bulmer, 1971). The gene frequencies in the next generation are given by

$$
\begin{gathered}
p_{1 i}^{*}=(1-m)\left(p_{1 i}+\Delta p_{1 i}\right)+m\left(p_{2 i}+\Delta p_{2 i}\right)=p_{1 i}+m\left(p_{2 i}-p_{1 i}\right) \\
+(1-m) \Delta p_{1 i}+m \Delta p_{2 i} \\
p_{2 i}^{*}=m\left(p_{1 i}+\Delta p_{1 i}\right)+(1-m)\left(p_{2 i}+\Delta p_{2 i}\right)=p_{2 i}+ \\
\left(\begin{array}{l}
\left(p_{1 i}-p_{2 i}\right) \\
+m \Delta p_{1 i}+(1-m) \Delta p_{2 i} .
\end{array}\right.
\end{gathered}
$$

At equilibrium $p_{1 i}^{*}=p_{1 i}$ and $p_{2 i}^{*}=p_{2 i}$, so that if $P_{1}$ and $P_{2}$ denote the equilibrium gene frequencies in the two populations, assumed to be the same at all loci, then

$$
\begin{aligned}
& m\left(P_{2}-P_{1}\right)+(1-m) \Delta P_{1}+m \Delta P_{2}=0 \\
& m\left(P_{1}-P_{2}\right)+m \Delta P_{1}+(1-m) \Delta P_{2}=0
\end{aligned}
$$

By addition,

$$
\Delta P_{1}+\Delta P_{2}=0
$$

By subtraction,

$$
(1-2 m)\left(\Delta P_{1}-\Delta P_{2}\right)=2 m\left(P_{1}-P_{2}\right) \text {. }
$$

We now suppose that $\frac{1}{2}\left(P_{1}+P_{2}\right)=P$ and $\frac{1}{2}\left(P_{1}-P_{2}\right)=\varepsilon$ so that $P_{1}=P$ $+\varepsilon, P_{2}=P-\varepsilon$. Without loss of generality it may be assumed that the mean value of $y$ is zero when $p_{i}=P$ at all loci, so that the mean values in the two populations at equilibrium are given by

$$
\begin{aligned}
& M_{1}=-2 \mathcal{N} a \varepsilon \\
& M_{2}=2 \mathcal{N} a \varepsilon
\end{aligned}
$$

from which it is clear that $\varepsilon$ is of order $1 / \sqrt{ } \mathcal{N}$ since $\mathcal{N} a$ is of order $\sqrt{ } \mathcal{N}$ and 
since $M_{1}$ and $M_{2}$ should be approximately independent of the number of loci. Hence

$$
\begin{aligned}
& P_{1} Q_{1}=(P+\varepsilon)(Q-\varepsilon)=P Q-\varepsilon(P-Q)-\varepsilon^{2} \\
& P_{2} Q_{2}=(P-\varepsilon)(Q+\varepsilon)=P Q+\varepsilon(P-Q)-\varepsilon^{2} .
\end{aligned}
$$

Substituting these expressions in equation 2 and ignoring terms smaller than $1 / \mathcal{N}$ we find that

$$
\begin{aligned}
& \Delta P_{1}=P Q\left\{A_{1} a-\frac{1}{2} B_{1} a^{2}(P-Q)\right\}-\varepsilon(P-Q) A_{1} a \\
& \Delta P_{2}=P Q\left\{A_{2} a-\frac{1}{2} B_{2} a^{2}(P-Q)\right\}+\varepsilon(P-Q) A_{2} a
\end{aligned}
$$

so that equations 5 and 6 can be re-written as

$$
\begin{aligned}
& P Q\left\{\left(A_{1}+A_{2}\right) a-\frac{1}{2}\left(B_{1}+B_{2}\right) a^{2}(P-Q)\right\}-\varepsilon a(P-Q)\left(A_{1}-A_{2}\right)=0 \\
& P Q\left\{\left(A_{1}-A_{2}\right) a-\frac{1}{2}\left(B_{1}-B_{2}\right) a^{2}(P-Q)\right\}-\varepsilon a(P-Q)\left(A_{1}+A_{2}\right)=4 m \varepsilon /(1-2 m) .
\end{aligned}
$$

It will also be convenient to define $V$ as the phenotypic variance and $h^{2} V$ as the genetic variance in a population in which $p_{i}=P$ at all loci, so that

$$
h^{2} V=2 \mathcal{N} a^{2} P Q \text {. }
$$

It follows from equation 8 that the actual genetic variance in either of the populations at equilibrium only differs from $h^{2} V$ by a quantity of order $1 / \sqrt{ } \mathcal{N}$.

We must now specify more precisely the selection pressures in the two populations. It will be supposed that both populations are subject to selection of the same intensity for an optimal value, $\theta$, but that $\theta$ takes different values, $\theta_{1}$ and $\theta_{2}$, in the two populations. As a mathematical model of selection for an optimal value we shall suppose that the fitness of an individual with phenotypic value $y$ is

$$
w(y)=\exp \left\{-c(y-\theta)^{2}\right\}
$$

where $c$ is a measure of the intensity of selecion, which will be assumed to be the same in the two populations. If $y$ is normally distributed, in a population with mean $\mu$ and variance $\sigma^{2}$, then it will also be normally distributed after selection with mean $\mu+D \mu$ and variance $\sigma^{2}+D \sigma^{2}$, where

$$
\begin{aligned}
& D \mu=2 c \sigma^{2}(\theta-\mu) /\left(1+2 c \sigma^{2}\right) \\
& D \sigma^{2}=-2 c \sigma^{4} /\left(1+2 c \sigma^{2}\right) .
\end{aligned}
$$

To prove this result we observe that the density function after selection is proportional to

$$
\begin{aligned}
& \exp -\left\{\frac{(y-\mu)^{2}}{2 \sigma^{2}}+c(y-\theta)^{2}\right\}= \\
& \exp -\left\{\frac{\left(1+2 c \sigma^{2}\right)}{2 \sigma^{2}}\left[y-\frac{\left(\mu+2 c \sigma^{2} \theta\right)}{\left(1+2 c \sigma^{2}\right)}\right]^{2}+\text { terms not involving } y\right\}
\end{aligned}
$$

which is the normal density function with the required mean and variance. In fact $y$ is not exactly normal, but the departure from normality is of order $1 / \sqrt{ } \mathcal{N}$, which is the order of the skewness of the genetic component of $y$. To the same order of approximation we may suppose that the variance in both populations is equal to $V$, as defined in the previous paragraph. To 
order 1 we may therefore write the changes in the mean and variance in the two populations as a result of selection as

$$
\begin{aligned}
& D M_{1}=k\left(\theta_{1}-M_{1}\right) \\
& D M_{2}=k\left(\theta_{2}-M_{2}\right) \\
& D V_{1}=D V_{2}=D V=-k V
\end{aligned}
$$

where

$$
k=2 c V /(1+2 c V) .
$$

We are now in a position to evaluate the coefficients $A_{1}, A_{2}, B_{1}$ and $B_{2}$ at equilibrium. It follows from the results of a previous paper (Bulmer, 1971) that $A_{1}$ and $B_{1}$ are, to order 1, given by

$$
\begin{aligned}
& A_{1}=-D M_{1} / V \\
& B_{1}=D V / V^{2}+A_{1}^{2}
\end{aligned}
$$

with similar results for $A_{2}$ and $B_{2}$. To order $1 / \sqrt{ } \mathcal{N}$ we may re-write equation 10 as

$$
P Q\left(A_{1}+A_{2}\right) a=0
$$

so that, to order 1 ,

$$
\begin{aligned}
& A_{2}=-A_{1} \\
& B_{1}=B_{2} .
\end{aligned}
$$

Hence equation 11 may be re-written to order $1 / \sqrt{ } \mathcal{N}$ as

$$
2 P Q A_{1} a=4 m \varepsilon /(1-2 m) \text {. }
$$

From equations $7,12,15,17$ and 20 we find that, to order 1 ,

$$
\begin{aligned}
M_{1} & =\frac{h^{2} k(1-2 m) \theta_{1}}{h^{2} k(1-2 m)+2 m} \\
A_{1} & =\frac{-2 m k \theta_{1}}{\left\{h^{2} k(1-2 m)+2 m\right\} V} .
\end{aligned}
$$

Finally let us define

$$
\begin{aligned}
A & \equiv \frac{-2 m k \theta_{1}}{\left\{h^{2} k(1-2 m)+2 m\right\} V} \\
B & =-k / V+A^{2} .
\end{aligned}
$$

Then, to order 1 ,

$$
\begin{aligned}
& A_{1}=A \\
& A_{2}=-A \\
& B_{1}=B_{2}=B .
\end{aligned}
$$

Since $B_{1}$ and $B_{2}$ are multiplied by $a^{2}$ in equation 2 it is only necessary to evaluate them to order 1 , so that this approximation is sufficiently accurate. However, $A_{1}$ and $A_{2}$ are multiplied by $a$ and must therefore be evaluated to order $1 / \mathcal{V} \mathcal{N}$. Let us therefore write

$$
\begin{aligned}
& A_{1}=A+\alpha_{1} \\
& A_{2}=-A+\alpha_{2}
\end{aligned}
$$


where $\alpha_{1}$ and $\alpha_{2}$ are small corrections of order $1 / \sqrt{ } \mathcal{N}$. From equation 10 it follows that, to order $1 / \mathcal{N}$,

$$
P Q\left\{\left(\alpha_{1}+\alpha_{2}\right) a-B a^{2}(P-Q)\right\}-2 \varepsilon a A(P-Q)=0
$$

which enables us to determine $\left(\alpha_{1}+\alpha_{2}\right)$ in terms of $\varepsilon$; it is fortunately unnecessary to determine $\alpha_{1}$ and $\alpha_{2}$ separately in order to determine the stability of the equilibrium. From equation 11 we can, to order 1 , write

$$
(1-2 m) h^{2} V A=4 m \varepsilon \mathcal{N} a
$$

which enables us to determine $\varepsilon$ to order $1 / \sqrt{ } \mathcal{N}$, which is sufficiently accurate.

So far it has been assumed that there is no dominance. If $D \neq 0$, then equation 2 must be replaced by

$$
\begin{array}{r}
\Delta p_{1 i}=p_{1 i} q_{1 i}\left\{A_{1} a\left[1+\left(p_{1 i}-q_{1 i}\right) D\right]-\frac{1}{2} B_{1} a^{2}\left[p_{1 i}-q_{1 i}\right)\left(1+\left(p_{1 i}-q_{1 i}\right) D\right)^{2}\right. \\
\left.\left.-4 p_{1 i} q_{1 i} D\right]\right\}
\end{array}
$$

with a corresponding expression for $\Delta p_{2 t}$ (Bulmer, 1971). Because of its complexity we shall only consider the symmetrical case where $P_{1}=\frac{1}{2}+\varepsilon$, $P_{2}=\frac{1}{2}-\varepsilon$ at equilibrium, so that $(P-Q)=0$. By the same argument as before it can be shown that, to sufficient accuracy, $B_{1}=B_{2}=B$ and that $A_{1}=A+\alpha_{1}, A_{2}=-A+\alpha_{2}$. An equation for $\left(\alpha_{1}+\alpha_{2}\right)$ is given by

$$
\left(\alpha_{1}+\alpha_{2}\right) a+4 A a \varepsilon D+B a^{2} D=0 .
$$

Equation 26 remains valid if $h^{2}$ is interpreted as the heritability in the strict sense, so that $h^{2} V$ is the additive genetic variance.

\section{The stability of The EQUilibrium}

To consider the stability of the equilibrium let us suppose that $p_{1 i}=P_{1}$ and $p_{2 i}=P_{2}$ at all loci, and that the gene frequencies are then perturbed by small quantities $e_{1 i}$ and $e_{2 i}$ respectively so that $p_{1 i}=P_{1}+e_{1 i}$ and $p_{2 i}+P_{2}$ $+e_{2 i}$. In the absence of dominance and in the symmetrical case where $P=Q=\frac{1}{2}$, it follows from equation 2 that

$$
\Delta p_{1 i}=\left(P_{1}+e_{1 i}\right)\left(Q_{1}-e_{1 i}\right)\left\{\left(A_{1}+\Delta A_{1}\right) a-B_{1} a^{2}\left(\varepsilon+e_{1 i}\right)\right\}
$$

where $\Delta A_{1}$ is the change in $A_{1}$ due to the change in the mean caused by the perturbation, which is given by

$$
\Delta A_{1}=\left(B_{1}-A_{1}^{2}\right) 2 a \Sigma e_{1 i} .
$$

(See Bulmer, 1971.) The equation for $\Delta p_{2 i}$ is obtained by changing the subscript throughout and substituting $-\varepsilon$ for $\varepsilon$. If we ignore terms of smaller magnitude than $1 / \mathcal{N}$, and if we also suppose that the perturbations are sufficiently small for their squares and products to be neglected, then

$$
\begin{aligned}
& \Delta p_{1 i}=\Delta P_{1}+\beta e_{1 i}+\gamma \Sigma e_{1 i} \\
& \Delta p_{1 i}=\Delta P_{2}+\beta e_{2 i}+\gamma \Sigma e_{2 i}
\end{aligned}
$$

where

$$
\begin{aligned}
& \beta=-\frac{1}{4} B a^{2}-2 \varepsilon A a \\
& \gamma=\frac{1}{2}\left(B-A^{2}\right) a^{2} .
\end{aligned}
$$


Furthermore, if $e_{1 i}^{*}$ and $e_{2 i}^{*}$ are the deviations from the equilibrium gene frequencies in the next generation, it follows from equation 3 that

$$
\begin{aligned}
& e_{1 i}^{*}=e_{1 i}+m\left(e_{2 i}-e_{1 i}\right)+(1-m)\left(\Delta p_{1 i}-\Delta P_{1}\right)+m\left(\Delta p_{2 i}-\Delta P_{2}\right) \\
& e_{2 i}^{*}=e_{2 i}+m\left(e_{1 i}-e_{2 i}\right)+m\left(\Delta p_{1 i}-\Delta P_{1}\right)+(1-m)\left(\Delta p_{2 i}-\Delta P_{2}\right) .
\end{aligned}
$$

Hence

$$
\begin{aligned}
& e_{1 i}^{*}=(1-m)(1+\beta) e_{1 i}+m(1+\beta) e_{2 i}+(1-m) \gamma \Sigma e_{1 i}+m \gamma \Sigma e_{2 i} \\
& e_{2 i}^{*}=m(1+\beta) e_{1 i}+(1-m)(1+\beta) e_{2 i}+m \gamma \Sigma e_{1 i}+(1-m) \gamma \Sigma e_{2 i} .
\end{aligned}
$$

Let us now write

$$
\begin{gathered}
s_{i}=e_{1 i}+e_{2 i} \\
d_{i}=e_{1 i}-e_{2 i} \\
\bar{s}=\Sigma s_{i} / \mathcal{N} \\
\bar{d}=\Sigma d_{i} / \mathcal{N}
\end{gathered}
$$

Then

$$
\begin{aligned}
\tilde{s}^{*} & =(1+\beta+\mathcal{N} \gamma) \bar{s} \\
\bar{d} & =(1-2 m)(1+\beta+\mathcal{N} \gamma) d \\
\left(s_{i}^{*}-\bar{s}^{*}\right) & =(1+\beta)\left(s_{i}-\bar{s}\right) \\
\left(d_{i}^{*}-\bar{d}^{*}\right) & =(1-2 m)(1+\beta)\left(d_{i}-\bar{d}\right) .
\end{aligned}
$$

The recurrence relationships for $\bar{s}$ and $d$ are dominated by the term $\left(1+\mathcal{N}_{\gamma}\right)$ $=\left(1-h^{2} k\right)$; since both $h^{2}$ and $k$ lie between 0 and 1 it follows that these quantities must be stable. The condition for the stability of the other two terms is that $\beta$ should be negative. Evaluating $\beta$ from the formulae given in the previous section, we find that the condition for the stability of the equilibrium is that

$$
\frac{h_{2} V}{2 \mathcal{N}}\left[\frac{k}{V}-A^{2} \frac{(1-m)}{m}\right]<0 .
$$

Since $\theta_{2}=-\theta_{1}$ from the suymmetry of the situation this finally gives the criterion for stability in the form

$$
\frac{\left(\theta_{1}-\theta_{2}\right)^{2}}{V}>\frac{\left\{h^{2} k(1-2 m)+2 m\right\}^{2}}{m(1-m) k} .
$$

Turning now to the asymmetrical case in which $P \neq Q$, but still assuming that there is no dominance, we find in the same way as before that, to order $1 / \mathcal{N}$,

Hence

$$
\begin{aligned}
& \Delta p_{1 i}=\Delta P_{1}+e_{1 i}\left(\beta-\delta+\zeta_{1}\right)+\gamma \Sigma e_{1 i} \\
& \Delta p_{2 i}=\Delta P_{2}+e_{2 i}\left(\beta+\delta+\zeta_{2}\right)+\gamma \Sigma e_{2 i} .
\end{aligned}
$$

$$
\begin{aligned}
\bar{s}^{*} & =\left\{1+\beta+\mathcal{N} \gamma+\frac{1}{2}\left(\zeta_{1}+\zeta_{2}\right)\right\} \bar{s}+\left\{-\delta+\frac{1}{2}\left(\zeta_{1}-\zeta_{2}\right)\right\} d \\
d^{*} & =(1-2 m)\left[\left\{1+\beta+\mathcal{N}_{\gamma}+\frac{1}{2}\left(\zeta_{1}+\zeta_{2}\right)\right\} \bar{d}+\left\{-\delta+\left(\zeta_{1}-\zeta_{2}\right)\right\} \bar{s}\right] \\
\left(s_{i}^{*}-\bar{s}^{*}\right) & =\left\{1+\beta+\frac{1}{2}\left(\zeta_{1}+\zeta_{2}\right)\right\}\left(s_{\dot{q}}-\bar{s}\right)+\left\{-\delta+\frac{1}{2}\left(\zeta_{1}-\zeta_{2}\right)\right\}(d-\bar{d}) \\
\left(d_{i}^{*}-\bar{d}^{*}\right) & =(1-2 m)\left[\left\{1+\beta+\frac{1}{2}\left(\zeta_{1}+\zeta_{2}\right)\right\}\left(d_{i}-\bar{d}\right)+\left\{-\delta+\frac{1}{2}\left(\zeta_{1}-\zeta_{2}\right)\right\}\left(s_{i}-\bar{s}\right)\right] .
\end{aligned}
$$


The constants in equations 39 and 40 are

$$
\begin{aligned}
& \beta=-P Q B a^{2}-2 \varepsilon A a+\frac{1}{2}(P-Q)^{2} B a^{2} \\
& \gamma=2 P Q a^{2}\left(B-A^{2}\right) \\
& \delta=(P-Q) A a \\
& \zeta_{1}=-(P-Q) \alpha_{1} a \\
& \zeta_{2}=-(P-Q) \alpha_{2} a .
\end{aligned}
$$

It is clear that the recurrence relationships for $\bar{s}$ and $d$ are dominated by the term $(1+\mathcal{N} \gamma)=\left(1-k h^{2}\right)$ which is certainly less than 1 in absolute value and thus ensures the stability of these terms. The stability of the terms $\left(s_{i}-\bar{s}\right)$ and $\left(d_{i}-\bar{d}\right)$ depends on the latent roots of the matrix

$$
\left[\begin{array}{cc}
1+\beta+\frac{1}{2}\left(\zeta_{1}+\zeta_{2}\right) & -\delta+\frac{1}{2}\left(\zeta_{1}-\zeta_{2}\right) \\
(1-2 m)\left\{-\delta+\frac{1}{2}\left(\zeta_{1}-\zeta_{2}\right)\right\} & (1-2 m)\left\{1+\beta+\frac{1}{2}\left(\zeta_{1}+\zeta_{2}\right)\right\}
\end{array}\right]
$$

The latent roots of this matrix are

$$
\begin{aligned}
\lambda=(1-m)\left\{1+\beta+\frac{1}{2}\left(\zeta_{1}+\zeta_{2}\right)\right\} & \pm\left[m^{2}\left\{1+\beta+\frac{1}{2}\left(\zeta_{1}+\zeta_{2}\right)\right\}^{2}\right. \\
& \left.+(1-2 m)\left\{-\delta+\frac{1}{2}\left(\zeta_{1}-\zeta_{2}\right)\right\}^{2}\right]^{\frac{1}{2}} .
\end{aligned}
$$

If we express the term after the \pm sign as $\left\{m^{2} x^{2}+(1-2 m) h\right\}^{\frac{1}{2}}$ and expand this expression in a Taylor series about $m^{2} x^{2}$, we find that

$$
\left\{m^{2} x^{2}+(1-2 m) h\right\}^{\frac{1}{2}}=m x+\frac{(1-2 m) h}{2 m x}+\ldots
$$

The dominant latent root is therefore

$$
\lambda_{1}=1+\beta+\frac{1}{2}\left(\zeta_{1}+\zeta_{2}\right)+\frac{(1-2 m)}{2 m} \delta^{2}+o(1 / \mathcal{N}) .
$$

The criterion for stability is therefore that

$$
\beta+\frac{1}{2}\left(\zeta_{1}+\zeta_{2}\right)+\frac{(1-2 m)}{2 m} \delta^{2}<0
$$

After some algebraic reduction this inequality gives

$$
\frac{h^{2} V}{2 \mathcal{N}}\left[\frac{k}{V}-A^{2} \frac{(1-m)}{m}\right]<0
$$

which leads to exactly the same criterion as before.

Finally, if the measure of dominance, $D$, is not zero, but if $P=Q=\frac{1}{2}$, we find in the same way as before that equations 39 and 40 are still valid if we define

$$
\begin{aligned}
\beta & =\frac{1}{4} B a^{2}-2 \varepsilon A a \\
\gamma & =\frac{1}{2} a^{2}\left(B-A^{2}\right) \\
\delta & =-\frac{1}{2} A a D \\
\zeta_{1} & =\frac{1}{2} \alpha_{1} a D \\
\zeta_{2} & =\frac{1}{2} \alpha_{2} a D .
\end{aligned}
$$

The criterion for stability given by equation 45 leads to

$$
\frac{h^{2} V}{2 \mathcal{N}}\left\{\left[\frac{k}{V}-\frac{(1-m)}{m} A^{2}\right]+D^{2}\left[\frac{k}{V}-A^{2}\right]\right\}<0
$$


If $m=\frac{1}{2}$ this leads to the same criterion as before, but if $m<\frac{1}{2}$ stability is more difficult to attain in the presence of dominance. If there is complete dominance, so that $D^{2}=1$, the criterion for stability is that

$$
\frac{\left(\theta_{1}-\theta_{2}\right)^{2}}{V}>\frac{2\left\{h^{2} k(1-2 m)+2 m\right\}^{2}}{m k} \text {. }
$$

Thus when $m$ is small the criterion for stability is twice as large as in the absence of dominance.

\section{Discussion}

The main conclusion of this paper is that if a metric character is subject to selection for different optimal clues, $\theta_{1}$ and $\theta_{2}$, in two populations, then a stable equilibrium is attained if

$$
\frac{\left(\theta_{1}-\theta_{2}\right)^{2}}{V}>C
$$

where $V$ is the phenotypic variance, and where the criterion of stability, $C$, is given in the absence of dominance by

$$
C=\frac{\left\{h^{2} k(1-2 m)+2 m\right\}^{2}}{m(1-m) k} .
$$

The criterion $C$ depends on $h^{2}$, the heritability of the character, $m$, the migration rate between the two populations, which lies between 0 for completely isolated populations and $\frac{1}{2}$ for populations which mix at random after selection, and $k$, a measure of the intensity of selection, which lies between 0 and 1 .

Numerical values of the criterion of stability for different values of $h^{2}$, $m$ and $k$ are shown in table 1. It will firstly be observed that if $m=\frac{1}{2}$, which corresponds to random mixture after selection, then $C=4 / k$. If the intensity of selection, $k$, is small then $C$ must be large so that a large difference between the optimal values is required to ensure stability; it is therefore unlikely that there will be stability in this case. It has been shown previously that in a single, panmictic population there can never be stability in the absence of dominance (Bulmer, 1971). The fact that it is possible, though unlikely, to attain stability under the present model with $m=\frac{1}{2}$ is analogous to the fact demonstrated by Levene (1953) that a stable equilibrium may exist at a single locus if there are two or more ecological niches without heterozygote advantage in any niche; the difference between the behaviour of a single population and of two populations with $m=\frac{1}{2}$ is due to the difference between the constant zygote number hypothesis and the constant fertile adult number hypothesis in the terminology of Dempster (1955).

When $m$ is small the stability criterion also becomes much smaller and takes the approximate value

$$
C=\left(h^{2} \alpha+2\right)^{2} / \alpha
$$

where $\alpha=k / m$. It will be noticed that, for fixed $\alpha, C$ is an increasing function of $h^{2}$. It follows that, if $\left(\theta_{1}-\theta_{2}\right)^{2} / V$ is greater than $C$ when $h^{2}=0$ (that is to say greater than $4 / \alpha$ ), then it will be equal to $C$ for some value of $h^{2}$ greater 
than 0 ; one would expect the heritability to be equal to this critical value at equilibrium. Solving the equation

$$
\frac{\left(\theta_{1}-\theta_{2}\right)^{2}}{V}=\left(h^{2} \alpha+2\right)^{2} / \alpha
$$

in terms of $h^{2}$ we therefore find that the heritability maintained by this mechanism is

$$
h^{2}=\frac{\left|\theta_{1}-\theta_{2}\right|}{\sqrt{ }(\alpha V)}-\frac{2}{a} .
$$

Furthermore, if we allow $\alpha$ to vary in equation 51 while $h^{2}$ remains constant,

TABLE 1

\begin{tabular}{|c|c|c|c|c|c|c|c|}
\hline \multicolumn{8}{|c|}{$h^{2}=0$} \\
\hline$k$ & $m$ & 0.01 & 0.02 & $0 \cdot 05$ & $0 \cdot 10$ & 0.20 & 0.50 \\
\hline 0.01 & & $4 \cdot 04$ & $8 \cdot 16$ & 21.05 & $44 \cdot 44$ & $100 \cdot 00$ & $400 \cdot 00$ \\
\hline 0.02 & & $2 \cdot 02$ & 4.08 & 10.53 & $22 \cdot 22$ & $50 \cdot 00$ & $200 \cdot 00$ \\
\hline 0.05 & & 0.81 & 1.63 & $4 \cdot 21$ & $8 \cdot 89$ & $20 \cdot 00$ & $80 \cdot 00$ \\
\hline $0 \cdot 10$ & & 0.40 & 0.82 & $2 \cdot 10$ & 4.44 & $10 \cdot 00$ & $40 \cdot 00$ \\
\hline $0 \cdot 20$ & & 0.20 & 0.41 & 1.05 & $2 \cdot 22$ & $5 \cdot 00$ & $20 \cdot 00$ \\
\hline 0.50 & & $0 \cdot 08$ & $0 \cdot 16$ & $0 \cdot 42$ & 0.89 & $2 \cdot 00$ & 8.00 \\
\hline 1.00 & & 0.04 & 0.08 & $0 \cdot 21$ & 0.44 & 1.00 & $4 \cdot 00$ \\
\hline
\end{tabular}

Values of the stability criterion, $\mathbf{C}$

\begin{tabular}{|c|c|c|c|c|c|c|}
\hline 0.01 & $6 \cdot 26$ & $10 \cdot 24$ & 22.99 & $46 \cdot 24$ & 101.51 & $400 \cdot 00$ \\
\hline $0 \cdot 02$ & 4.48 & $6 \cdot 28$ & 12.51 & $24 \cdot 04$ & 51.51 & $200 \cdot 00$ \\
\hline 0.05 & $4 \cdot 00$ & $4 \cdot 18$ & $6 \cdot 32$ & 10.76 & 21.53 & $80 \cdot 00$ \\
\hline $0 \cdot 10$ & $4 \cdot 81$ & 3.95 & $4 \cdot 43$ & $6 \cdot 40$ & 11.56 & 40.00 \\
\hline $0 \cdot 20$ & $7 \cdot 03$ & 4.72 & 3.80 & $4 \cdot 36$ & $6 \cdot 61$ & $20 \cdot 00$ \\
\hline 0.50 & $14 \cdot 19$ & 8.00 & 4.45 & 3.56 & 3.78 & 8.00 \\
\hline 1.00 & $26 \cdot 27$ & $13 \cdot 80$ & $6 \cdot 37$ & 4.00 & 3.06 & $4 \cdot 00$ \\
\hline \multicolumn{7}{|c|}{$h^{2}=1$} \\
\hline$k$ & $m \quad 0.01$ & 0.02 & 0.05 & $0 \cdot 10$ & 0.20 & 0.50 \\
\hline 0.01 & 8.97 & 12.55 & $25 \cdot 01$ & 48.07 & 103.02 & $400 \cdot 00$ \\
\hline 0.02 & 7.92 & 8.94 & 14.66 & 25.92 & 53.04 & $200 \cdot 00$ \\
\hline 0.05 & $9 \cdot 62$ & $7 \cdot 90$ & $8 \cdot 85$ & $12 \cdot 80$ & $23 \cdot 11$ & $80 \cdot 00$ \\
\hline $0 \cdot 10$ & $14 \cdot 06$ & 9.44 & $7 \cdot 60$ & 8.71 & $13 \cdot 22$ & $40 \cdot 00$ \\
\hline $0 \cdot 20$ & 23.56 & 13.73 & $8 \cdot 25$ & $7 \cdot 20$ & 8.45 & $20 \cdot 00$ \\
\hline 0.50 & 52.54 & 27.59 & 12.74 & 8.00 & $6 \cdot 12$ & 8.00 \\
\hline 1.00 & 101.01 & 51.02 & $21 \cdot 05$ & $11 \cdot 11$ & $6 \cdot 25$ & 4.00 \\
\hline
\end{tabular}

$\begin{array}{lllllll} & m & 0.01 & 0.02 & h^{2}=\frac{1}{2} & & \\ k & & & 0.05 & 0.10 & 0.20 & 0.50\end{array}$

the minimum value which $C$ can take is $8 h^{2}$; this value is attained at $\alpha=2 / h^{2}$. The maximum heritability which can be maintained is therefore $\left(\theta_{1}-\theta_{2}\right)^{2} / 8 \mathrm{~V}$. This model therefore provides a mechanism for the maintenance of genetic variability in natural populations, and predicts the amount of genetic variability to be expected.

For many metric characters which have been investigated the heritability is about $\frac{1}{2}$, and in this case equation 50 becomes

$$
C=\left(\frac{1}{2} \alpha+2\right)^{2} / \alpha
$$


which has a minimum value of 4 at $\alpha=4$. It will be seen from table 1 that, when $h^{2}=\frac{1}{2}$, the stability criterion can take a value as low as 4 for quite a wide range of values of $m$ and $k$. If $C$ is approximately equal to 4 , then the requirement for stability is that the difference between the two optimal values is larger than 2 standard deviations. The difference between the observed mean values in the two populations at equilibrium will be considerably smaller than the difference between the optimal values, as indicated by equation 21 ; if $m$ is small then

$$
\left(M_{1}-M_{2}\right)=\frac{h^{2} \alpha}{\left(2+h^{2} \alpha\right)}\left(\theta_{1}-\theta_{2}\right) .
$$

Thus if $h^{2}=\frac{1}{2}$ and $\alpha=4$, then the difference between the means will be only one standard deviation when the difference between the optimal values is 2 standard deviations. If $h^{2}=\frac{1}{2}$ and $\alpha=1$, it is necessary to postulate a difference of $2 \frac{1}{2}$ standard deviations between the optimal values to ensure stability, but this would only be accompanied by a difference of half a standard deviation between the means. It does not seem unreasonable to postulate a difference of this order of magnitude, and it is therefore suggested that partial geographic isolation, with different ecological requirements in the different geographical areas (or ecological niches), may play an important role in maintaining genetic variability in natural populations. Such a mechanism is in accordance with modern ideas about the population structure of species as described, for example, by Mayr (1966). To make the model more realistic, and in particular to construct a model of a continuous geographic cline, it would be desirable to extend it to a larger number of partially isolated populations and to populations distributed continuously in space with isolation by distance. It is hoped to do this in another paper.

\section{Summary}

1. A model is formulated to describe the behaviour of a metric character subject to selection for different optimal values in two populations.

2. If $\theta_{1}$ and $\theta_{2}$ are the two optimal values and $V$ the phenotypic variance, it is shown that a stable equilibrium will exist if $\left(\theta_{1}-\theta_{2}\right)^{2} / V$ is greater than a critical value, $C$, which depends on the heritability of the character, the intensity of selection and the migration rate between the two populations.

3. It is suggested that this mechanism may play an important part in the maintenance of genetic variability in natural populations.

\section{REFERENGES}

Bulmer, M. G. 1971. The stability of equilibria under selection. Heredity, 27, 157-162. Dempster, E. R. 1955. Maintenance of genetic heterogeneity. Cold Spring Harbor Symp. Quant. Biol., 20, 25-32.

LEVENE, H. 1953. Genetic equilibrium when more than one ecological niche is available. Amer. Nat., 87, 331-333.

LEVEne, H. 1967. Genetic diversity and diversity of environment: mathematical aspects. Proceedings of the Fifth Berkeley Symposium on Mathematical Statistics and Probability, 4, 305316.

MAYr, E. 1966. Animal Species and Evolution. Harvard University Press, Cambridge, Mass. Moran, P. A. P. 1962. Statistical Processes of Evolutionary Theory. Clarendon Press, Oxford. 